\title{
LONG-TERM UNDIAGNOSED DERMATOMYOSITIS WITH DISTAL WEAKNESS AND URINARY RETENTION: IS IT ANTI-NXP2 PHENOTYPE?
}

Erika Biegelmeyer ${ }^{1, \star}$, Evelise Mileski do Amaral Berlet ${ }^{1}$, Julia Boechat Farani ${ }^{1}$, Marília Voges de Souza ${ }^{1}$, Marcel Mathias Villaça ${ }^{1}$, Mauro Waldemar Keiserman ${ }^{1}$

1.Pontifícia Universidade Católica do Rio Grande do Sul, Porto Alegre (RS), Brazil.

*Corresponding author: erika.biegel@gmail.com

\section{BACKGROUND}

Dermatomyositis is defined by typical cutaneous presentations and proximal weakness, eventually associated to calcinosis cutis. When associated with antibody anti-NXP2, it can present a severe disease with distal weakness, dysphagia and a calcinosis cutis.

\section{CASE REPORT}

A 41-year-old woman was hospitalized due to an 18-month history of erythematous rash, proximal and distal weakness, dysphagia and a recent urinary retention and constipation. She had previously been empirically treated with $60 \mathrm{mg}$ of prednisone on general medical care, with resolution of skin lesions, but few improvements of muscle weakness. The lesions resurfaced and worsened after discontinuation of treatment. She also reported fatigue, a $40 \mathrm{~kg}$ weight loss, Raynaud phenomenon initiated after the beginning of skin lesions and painful nodules on armpits since the first symptoms. On physical examination she had hyperpigmented papules on the forehead, cheeks and neck, dyschromic papules and poikiloderma of the extensor surface of the upper arms and the lateral thigh, this last one consistent with the Holster sign and Gottron sign on elbows. There was painful calcinosis cutis on armpits (also seen on CT scan), antecubital fossa, chest, inframammary, dorsal and gluteal regions. The muscle strength in the deltoid, quadriceps and neck were 2 out of 5 , whilst wrist-extensors and ankle-dorsiflexors were 4 . MMT8 was 30 out of 80 . She had high inflammatory tests, normal $\mathrm{CK}$, aldolase, lactate dehydrogenase and transaminases. A positive ANA 1/5120 with multiple nuclear dots pattern (AC-6). Thighs MRI had proximal muscle inflammation and diffuse and intense subcutaneous edema. We diagnosed dermatomyositis with calcinosis cutis, compatible with anti-NXP2 antibody. Unfortunately, there were no resources to dosage this antibody. Malignancy investigation was negative. The patient was treated with corticosteroid and azathioprine, slowly improving weakness and urinary retention, besides resolution of skin dyschromia. Diltiazem and bisphosphonate were initiated for calcinosis and we plan to associate rituximab on outpatient clinic.

\section{CONCLUSION}

We hereby present a probable NXP2 phenotype of dermatomyositis, with long-term evolution of a severe disease with calcinosis, chronic rash, proximal and distal weakness, a compatible ANA and ultimately a urinary retention. We believe the urinary retention was due to detrusor weakness related to long-term untreated disease. The normal $\mathrm{CK}$ was probably explained by muscle consumption, that also predicts a slowly and perhaps incomplete recovery. Considering strong malignancy association, we plan on performing serial screening on outpatient scenario.

\section{KEYWORDS}

Inflammatory myopathies, Anti-NXP2, Calcinosis cutis, Distal weakness. 